\title{
Tratamiento endovascular de aneurisma aórtico abdominal: resultados en 80 pacientes consecutivos
}

\author{
Francisco Valdés $\mathrm{E}^{1}$, Renato Mertens $\mathrm{M}^{1}$, \\ Albrecht Krämer Sch ${ }^{1}$, Michel Bergoeing $\mathbf{R}^{1}$, \\ Leopoldo Mariné $M^{1}$, Roberto Canessa $B^{2}$, \\ Alvaro H uete $\mathbf{G}^{3}$, Jeanette Vergara $\mathbf{G}^{\mathrm{la}}$, \\ Magaly Valdebenito C ${ }^{1 a}$, Dixiana Rivera $D^{4, a}$. \\ Endovascular repair of abdominal \\ aortic aneurysm. Results in 80 \\ consecutive patients
}

Background: Endovascular repair of abdominal aortic aneurysms (AAA) avoids laparotomy, shortens hospital stay and reduces morbidity and mortality related to surgical repair, allowing full patient recovery in less time. Aim: To report short and long term results of endovascular repair of AAA in 80 consecutive patients treated at our institution. Patients and Methods: Between September 1997 and February 2005, three women and 77 men with a mean age $73.6 \pm 7.7$ years with AAA $5.8 \pm 1.0 \mathrm{~cm}$ in diameter, were treated. The surgical risk of $38 \%$ of patients was grade III according to the American Society of Anesthesiologists classification. Each procedure was performed in the operating room, under local or regional anesthesia, with the aid of digital substraction angiography. The endograft was deployed through the femoral artery (83.7\% bifurcated, $16.3 \%$ tubular graft). A femoro-femoral bypass was required in $11.3 \%$ of cases. Follow-up included a spiral CT scan at 1, 6 and 12 months postoperatively, and then annually. Results: Endovascular repair was successfully completed in 79/80 patients (98.7\% technical success). The procedures lasted $147 \pm 71 \mathrm{~min}$. Length of stay in the observation unit was $20.6 \pm 13.5 \mathrm{~h}$. Blood transfusion was required in $10 \%$. Sixty two percent of the patients were discharged before $72 \mathrm{~h}$. One patient died 8 days after surgery due to a myocardial infarction (1.3\%). During follow-up (3-90 months), 1 patient developed late AAA enlargement due to a type I endoleak, requiring a new endograft. No AAA rupture was observed. Survival at 4 years was $84.2 \%$ ( $\mathrm{SE}=9.2$ ). Endovascular re-intervention free survival was 82.7\% ( $\mathrm{SE}=9.5$ ). Conclusion: Endovascular surgery allows effective exclusion of AAA avoiding progressive enlargement and/or rupture and is a good alternative to open repair. Close and frequent postoperative follow up is mandatory (Rev Méd Chile 2006; 134: 1265-74).

(Key words: Aortic aneurysm; Blood vessel prosthesis)

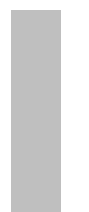

\footnotetext{
Recibido el 11 de octubre, 2005. Aceptado el 3 de abril, 2006.

${ }^{1}$ Cirugía Vascular y Endovascular, Departamento de Enfermedades Cardiovasculares y División de Cirugía. ${ }^{2}$ Anestesia Cardiovascular, Departamento de Anestesiología. ${ }^{3}$ Imagenología Cardiovascular, Departamento de Radiología, Facultad de Medicina y Hospital Clínico, Pontificia Universidad Católica de Chile. ${ }^{4}$ Instituto Vascular de Santiago.

aEnfermera Universitaria
}

Correspondencia a: Dr. Francisco Valdés E. Cirugía Vascular y Endovascular, Hospital Clínico y Facultad de Medicina, Pontificia Universidad Católica de Chile. Marcoleta 367 6o piso. Fono (56-2) 686-3268. Fax (56-2) 632 6812. E mail: franval@med.puc.cl 
$E^{n}$ 1990, Parodi desarrolló y aplicó exitosamente una técnica mínimamente invasiva para el tratamiento del aneurisma aórtico abdominal (AAA), en la que insertando una prótesis a través de las arterias femorales, se excluye efectivamente el AAA de la circulación, se evita una laparotomía y se elimina el riesgo de ruptura ${ }^{1}$. Desde entonces, dicha técnica se ha perfeccionado, difundido universalmente, y transformado rápidamente en una alternativa para pacientes que presentan una anatomía compatible, especialmente si son considerados de alto riesgo para la cirugía convencional $^{2,3}$. Nuestra experiencia inicial con esta técnica fue reportada en $1998^{4}$.

El objetivo del presente trabajo es analizar la efectividad del tratamiento del AAA por vía endovascular, su morbilidad, durabilidad y la sobrevida alejada en 80 pacientes consecutivos tratados en nuestro hospital.

\section{PACIENTES Y MÉTOdOS}

Se incluyeron todos los pacientes tratados de AAA por vía endovascular en nuestro Hospital Clínico, a partir de septiembre de 1997, los cuales han sido incorporados a un registro computacional prospectivo. Se excluyeron 50 pacientes tratados en el período con similar técnica, por aneurismas, trauma o disección de la aorta torácica, o lesiones aisladas de las arterias ilíacas.
Sólo tratamos pacientes cuyo AAA cumpliera con los requisitos anatómicos recomendados, analizados en un estudio de imágenes adecuado (Tabla 1). Para tal efecto, en los primeros casos se efectuó una aortografía abdominal biplanar con catéter graduado en centímetros (Figura 1) y, a partir del año 2000, se realizó un escáner helicoidal en fase arterial (Figura 2). Se realizó la evaluación clínica y los estudios de laboratorio preoperatorios habituales para cirugía de la aorta abdominal.

Las endoprótesis utilizadas se detallan en la Tabla 2. Su diseño es de tipo modular. Aquellas bifurcadas se componen de un cuerpo principal aorto-ilíaco unilateral $y$, en forma separada, una rama para la arteria ilíaca contralateral, que se acopla al segmento anterior. En algunos casos utilizamos una prótesis tubular, conformada por un segmento aorto-uni-ilíaco, requiriendo la construcción de un puente extraanatómico fémorofemoral para revascularizar la extremidad contralateral. A partir del año 2000, hemos utilizado preferentemente una endoprótesis trimodular de fijación suprarrenal (Zenith ${ }^{\circledR} \mathrm{COOK}^{\circledR}$, Bloomington, Ind., EE.UU).

La intervención se efectuó en pabellón quirúrgico bajo anestesia local o de preferencia regional, con la monitorización estándar para cirugía aórtica y con todas las condiciones exigidas para efectuar cirugía abierta, de ser ésta necesaria. Para la generación de imágenes intraoperatorias se usó un

\title{
Tabla 1. Requisitos anatómicos necesarios para la reparación endovascular del AAA
}

\author{
Cuello aórtico* (aorta sana bajo las arterias renales) \\ Longitud $\geq 1,5 \mathrm{~cm}$ \\ Diámetro $=1,8-2,8 \mathrm{~cm}$ \\ Angulación $<60^{\circ}$ \\ Ausencia de trombo mural o calcificaciones severas \\ Arterias Ilíacas comunes \\ Diámetro $\leq 2,0 \mathrm{~cm}$ \\ Ausencia de tortuosidad, calcificación o estenosis que impida el acceso vascular directo, o \\ por conducto ilíaco \\ Ausencia de ramas viscerales significativas, originadas del AAA \\ Arterias renales \\ Arteria mesentérica inferior compensatoria por oclusión de la mesentérica superior
}

*Las prótesis Talent ${ }^{\circledR}$ y Zenith ${ }^{\circledR}$ tienen una porción de stent descubierta, lo que permite su fijación por sobre las arterias renales. 
FIgURA 1. Aortografía por sustracción digital (antero-posterior y lateral) mediante catéter graduado en $\mathrm{cm}$, para medición exacta del largo y diámetro de los distintos segmentos de la aorta e ilíacas.

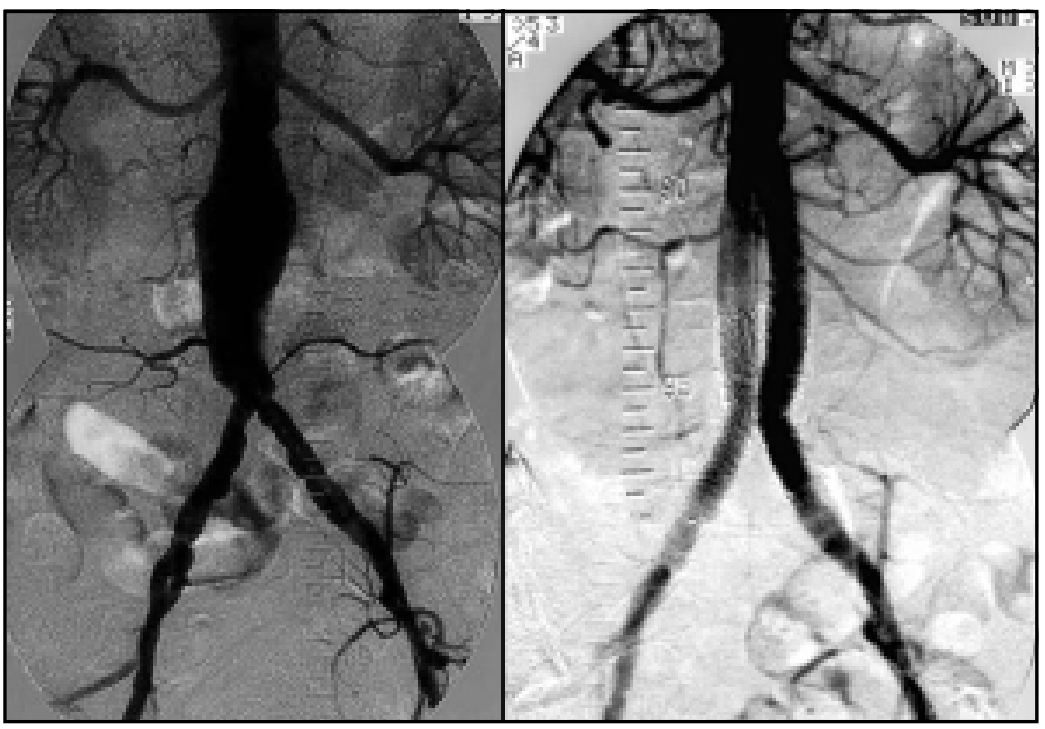

FiguRA 2. Escáner helicoidal: cortes transversales (izquierda) y reconstrucción sagital de AAA (derecha).

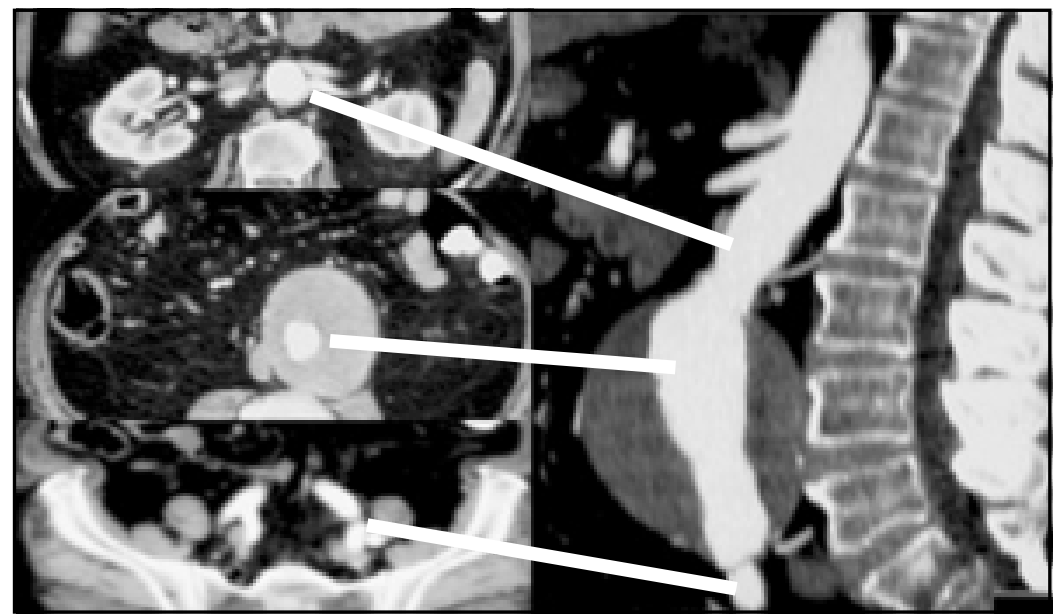

Tabla 2. Tipo de endoprótesis utilizados en la reparación de AAA 1997-2005 N =80 casos

\begin{tabular}{|lccrr|}
\hline Modelo & Fabricante & Sitio de fijación proximal & $\mathrm{N}$ & $\%$ \\
\hline Vanguard $^{\circledR}$ & Boston Scientific & Infrarrenal & 4 & 5,0 \\
Talent $^{\circledR}$ & Medtronic & Suprarrenal & 5 & 6,3 \\
Excluder $^{\circledR}$ & Gore & Infrarrenal & 3 & 3,8 \\
Zenith $^{\circledR}$ & Cook & Suprarrenal & 68 & 85,0 \\
\hline
\end{tabular}


equipo portátil de angiografía por sustracción digital Siemens ${ }^{\circledR}$, modelo Siremobil 2000 (Erlangen, Alemania), y a partir del año 2002, un angiógrafo OEC 9800 General Electric ${ }^{\circledR}$, (Utah, EE.UU).

El implante de la endoprótesis se realizó por vía transfemoral, siendo necesaria la exposición quirúrgica de la arteria en forma uni o bilateral según el dispositivo utilizado. El despliegue y ensamblaje de la endoprótesis se realizó bajo control radioscópico. El protocolo contempla una permanencia transitoria en recuperación posoperatoria y el alta hospitalaria dentro de las $72 \mathrm{~h}$. Posteriormente, el paciente es controlado con un escáner helicoidal dentro del mes, a los 6 y 12 meses y luego anualmente. Los pacientes fueron seguidos clínicamente en forma personal por el cirujano, por el médico referente o por teléfono. En caso de fallecimiento, se obtuvo además el certificado de defunción del Registro Civil.

Para el análisis de los resultados se usó el test de Student pareado y para la evaluación de la sobrevida las tablas de Kaplan-Meier, siguiendo las recomendaciones de la Sociedad de Cirugía Vascular de Norteamérica ${ }^{5}$.

\section{RESULTADOS}

Durante el periodo septiembre 1997-febrero 2005, tratamos 352 pacientes por AAA, empleando esta nueva técnica en 80 (22,7\%). Las características clínicas de los pacientes se resumen en la Tabla 3, destacando entre ellas una alta prevalencia de enfermedad coronaria. El 61,2\% tenía riesgo ASA II y $38,8 \%$ ASA III. El diámetro promedio del AAA fue $5,86 \pm 1,04 \mathrm{~cm}$. Cinco pacientes $(6,3 \%)$ habían tenido cirugía aórtica entre 3 y 25 años antes y desarrollaron un aneurisma aórtico anastomótico, que motivó la reparación endovascular.

El primer caso de la serie debió ser convertido a cirugía abierta (puente aorto bi-ilíaco convencional) por dificultad técnica durante el ensamblaje

Tabla 3. Características clínicas y demográficas de 80 pacientes tratados por AAA con endoprótesis aórtica, septiembre 1997-febrero 2005

\begin{tabular}{|llrr|}
\hline Variable & & $\mathrm{N}$ & $\%$ \\
\hline \multirow{2}{*}{ Edad } & 52-91 años $(73,6 \pm 7,7)$ & & \\
Patologías & Masculino & & \\
& Femenino & 77 & 96,2 \\
& Aneurisma ilíaco & 3 & 3,8 \\
Aneurisma aórtico inflamatorio & 6 & 7,5 \\
Cirugía aórtica previa & 4 & 5,0 \\
Puente fémoro-femoral previo & 5 & 6,3 \\
Insuficiencia arterial de extremidades inferiores & 3 & 3,8 \\
Cardiopatía coronariał & 8 & 10,0 \\
Valvulopatía aórtica & 52 & 65,0 \\
Arritmia & 3 & 3,8 \\
Hipertensión arterial & 7 & 8,7 \\
Tabaquismo crónico & 40 & 50,0 \\
Enfermedad pulmonar obstructiva crónica & 19 & 23,8 \\
Dislipidemia & 24 & 30,0 \\
Diabetes Mellitus & 15 & 18,8 \\
Obesidad & 9 & 11,3 \\
Neoplasia operada (próstata, colon) & 8 & 10,0 \\
Abdomen hostil & 13 & 16,3 \\
& 2 & 2,5 \\
\hline
\end{tabular}

t: Incluye 16 pacientes (20\%), con revascularización coronaria previa. 
FIGURA 3. Izquierda: reconstrucción tridimensional preprocedimiento. Derecha: reconstrucción tridimensional posprocedimiento.
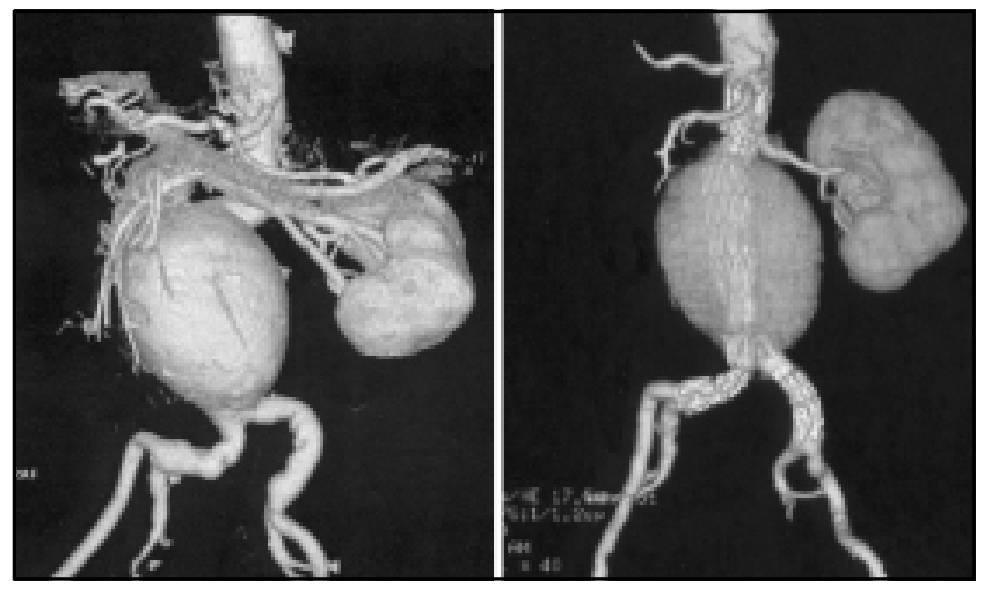

del injerto. En los 79 casos restantes, se completó el implante en forma exitosa (éxito técnico 98,8\%), logrando la exclusión completa del AAA (Figura 3). Los procedimientos efectuados se detallan en la Tabla 4.

La evolución posoperatoria intrahospitalaria se resume en la Tabla 5, destacando un descenso del hematocrito de 7,39 $\pm 4,1 \%$ (IC 95\%, p <0,0001). Sólo 10\% (8 casos) requirió transfusión de glóbulos rojos. La creatinina plasmática no mostró variación significativa. El $56 \%$ de los pacientes presentó alza térmica sobre $37,5^{\circ} \mathrm{C}, 34 \%$ presentó leucocitosis mayor de $10.000 / \mathrm{mm}^{3}$, y $76 \%$ tuvo una VHS mayor de $20 \mathrm{~mm} / \mathrm{hr}$.

El seguimiento se extendió entre 3 y 90 meses, habiéndose perdido de control sólo dos pacientes $(2,5 \%)$, a los 22 y 72 meses. Un paciente $(1,3 \%)$ falleció antes de los 30 días en su domicilio, a
Tabla 4. Procedimientos efectuados para la reparación endovascular de AAA en 80 casos

\begin{tabular}{|lrr|}
\hline Procedimiento & N & $\%$ \\
\hline Prótesis bifurcada & 67 & 83,7 \\
Prótesis aorto uni ilíaca & 12 & 15,0 \\
Prótesis aorto-aórtica tubular & 1 & 1,3 \\
Conducto retroperitoneal & 2 & 2,5 \\
Puente fémoro-femoral & 9 & 11,3 \\
Oclusor uni ilíaco & 3 & 3,8 \\
Stent ilíaco & 2 & 2,5 \\
Extensión de rama & 9 & 11,3 \\
Embolización hipogástrica unilateral & 8 & 10,0 \\
Stent renal por estenosis renal & 2 & 2,5 \\
Conversión a cirugía abierta & 1 & 1,3 \\
& & \\
\hline
\end{tabular}

Tabla 5. Evolución intrahospitalaria posimplante de endoprótesis aórtica abdominal

\begin{tabular}{|lll|}
\hline Duración de la intervención & $($ minutos $)$ & $147 \pm 72$ \\
Permanencia en recuperación & $(\mathrm{h})$ & $20,6 \pm 13,5$ \\
Permanencia posoperatoria & $(\mathrm{h})$ & $75,6 \pm 42,5$ \\
Temperatura máxima posoperatoria & $\left({ }^{\circ} \mathrm{C}\right)$ & $37,4 \pm 0,8$ \\
Variación del hematocrito & $(\%, 95 \% \mathrm{IC})$ & $-7,39(-8,42 \mathrm{a}-6,36) \mathrm{p}<0,0001$ \\
Recuento de leucocitos posoperatorio & $\left(\mathrm{cel} / \mathrm{mm}^{3}\right)$ & $9.696 \pm 2.500$ \\
Velocidad de sedimentación & $(\mathrm{mm} / \mathrm{hr})$ & $43 \pm 27$ \\
Variación de la creatinina & $(\mathrm{mg} \%, 95 \%$ IC) & $0,056(-0,03$ a 0,14$) \mathrm{p}=\mathrm{NS}$ \\
Transfusión posoperatoria & $(\%$ de pacientes $)$ & 10 \\
\hline
\end{tabular}


FIgURA 4. Escáner helicoidal. Izquierda: corte transversal al mes de efectuado el implante protésico que demuestra aneurisma trombosado, ausencia de endofiltración y ramas de endoprótesis con flujo normal. Derecha: estudio efectuado a los 44 meses, confirmando exclusión completa y desaparición del saco aneurismático.

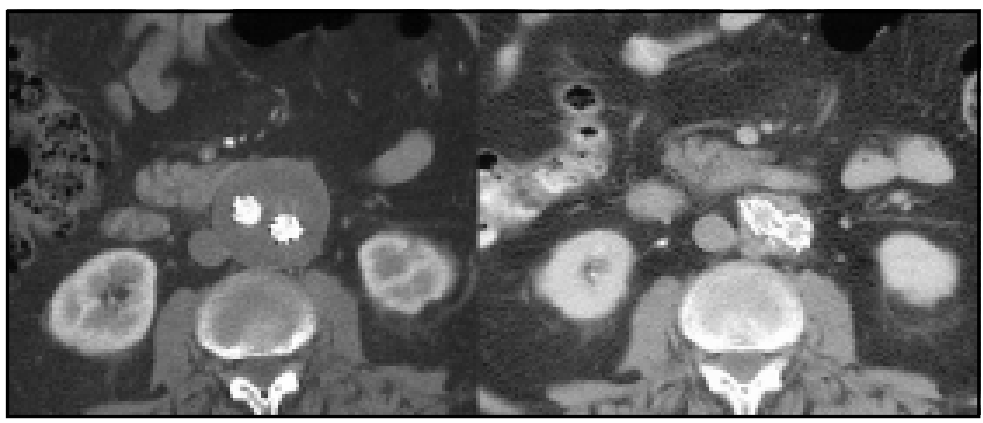

causa de un infarto del miocardio. Durante el seguimiento, 5 pacientes presentaron complicaciones relacionadas con la endoprótesis. En 2 casos se ocluyó una rama ilíaca: a los 2 y 84 meses, requiriendo la construcción de un puente fémoro-femoral sólo el caso más tardío. Un caso, a los 48 meses, presentó migración distal de la endoprótesis (Vanguard ${ }^{\circledR}$, Boston Scientific, Natick, Ma. EE.UU), con endofiltración proximal tipo 1 y reaparición de un AAA de 7,2 cm, el que se excluyó exitosamente con una nueva endoprótesis. En otro caso, a los 39 meses se advirtió un deslizamiento parcial de una rama ilíaca (Zenith ${ }^{\circledR}$ ) sin endofiltración, siendo tratado profilácticamente por vía endovascular con una extensión de la endoprótesis. Un paciente, a los 33 meses de seguimiento, presentó oclusión en forma aguda de su endoprótesis aorto uni-ilíaca, cursando el posoperatorio, inmediato de un reemplazo total de cadera, siendo resuelto de emergencia con un puente axilo femoral convencional.

En un paciente, el escáner de control al primer año demostró gas periprotésico, sospechándose una infección. Al momento de este reporte se encontraba asintomático, con desaparición del AAA, completando 48 meses de seguimiento, con terapia antibiótica a permanencia como único tratamiento.

El 98\% de los pacientes con más de 12 meses de operados mostró mantención o reducción del diámetro del AAA. (Figuras 4 y 5). La sobrevida a 5 años libre de ruptura aórtica fue 100\% $(E S=4,5)$, la sobrevida a 4 años libre de complicaciones relacionadas con el dispositivo fue $82,7 \%$ (ES=9,5), y la sobrevida general a 4 años $84,2, \%$ $(E S=9,2)$ (Figura 6). Seis pacientes han fallecido durante el seguimiento posoperatorio alejado,

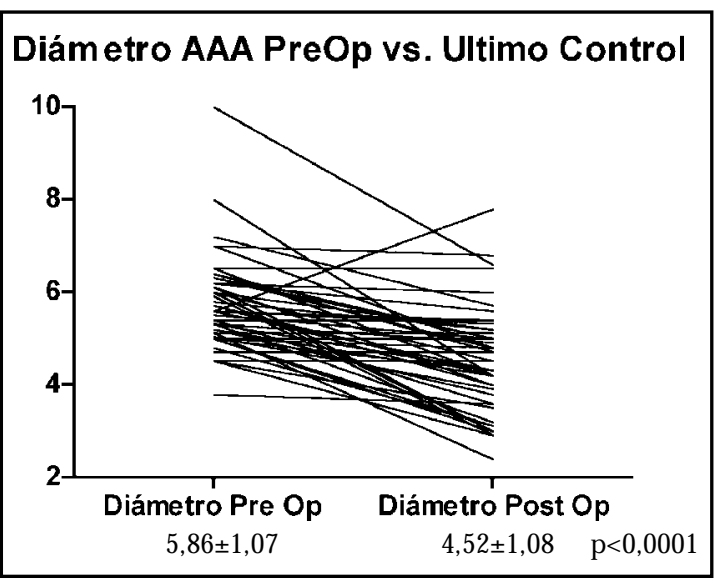

FiguRA 5. Reducción del diámetro del AAA observada entre el escáner preoperatorio y el último escáner de control.

entre los 3,5 y los 52 meses (promedio 25,8 meses, Tabla 6).

En la Tabla 7 se comparan los resultados observados en nuestros pacientes con series recientemente publicadas.

\section{DisCUSIÓN}

La incidencia de AAA en Chile es similar a la comunicada en países del hemisferio norte ${ }^{6}$, con aumento progresivo sobre los 65 años, edad en que los riesgos operatorios son objetivamente mayores. La indicación de reparación del AAA se basa en el riesgo de ruptura y muerte, evento que tiene directa relación con el diámetro de la aorta. 


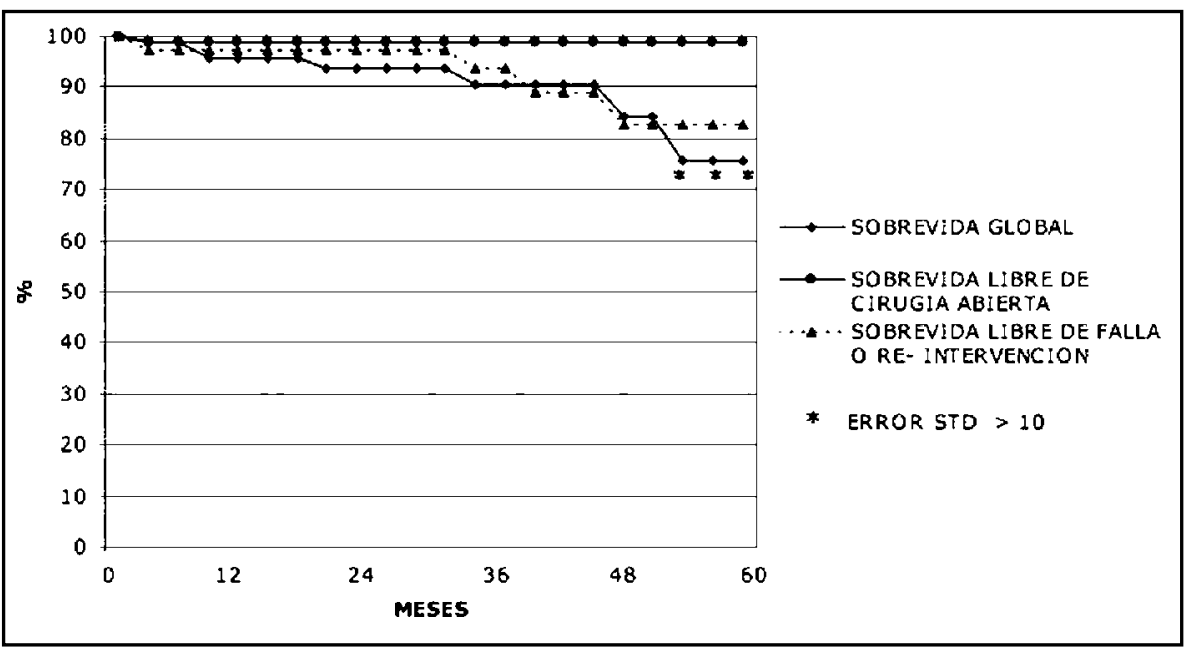

FIguRA 6. Tabla de sobrevida actuarial a 5 años (Kaplan Meier).

Según estudios efectuados en poblaciones cerradas, la mortalidad por ruptura de AAA es $80-90 \%$ si se incluyen la mortalidad prehospitalaria y la mortalidad intrahospitalaria, pre y posoperatoria ${ }^{7}$.

En base a la evidencia disponible a la fecha, el diámetro crítico para indicar una intervención electiva de AAA en el spaciente promedio»sería 5,5 $\mathrm{cm}^{8,9}$. Sin embargo, nuestro criterio, compartido por otros investigadores, es evaluar la posibilidad de reparación a partir de los $4,5 \mathrm{~cm}$, en especial en pacientes jóvenes o de menos riesgo para una intervención, en pacientes de sexo femenino o en casos con alto riesgo de ruptura, y también en pacientes residentes en áreas alejadas, con dificultad de acceso a un centro hospitalario capacitado en tratar esta patología con resultados operatorios dentro de los estándares contemporáneos. Por el contrario, postergar la reparación más allá de los
Tabla 6. C ausas de mortalidad alejada posreparación endovascular de AAA

\begin{tabular}{|c|c|}
\hline Causa & $\mathrm{N}$ \\
\hline * Neumopatía aguda & 2 \\
\hline * Insuficiencia cardíaca & 1 \\
\hline * Infarto del miocardio & 1 \\
\hline * Infarto cerebral & 1 \\
\hline * Desnutrición & 1 \\
\hline
\end{tabular}

5,5 cm de diámetro puede ser una opción válida en pacientes de muy alto riesgo, en especial si la anatomía de su AAA es desfavorable para un tratamiento endovascular ${ }^{10-12}$. Los AAA tratados con esta técnica en la presente comunicación tuvieron un diámetro de 5,86 $\mathrm{cm}$ en promedio.

Tabla 7. Resultados del tratamiento endovascular del AAA

\begin{tabular}{|lcccccc|}
\hline Autor & Año & N & $\begin{array}{c}\text { Éxito } \\
\%\end{array}$ & $\begin{array}{c}\text { Diámetro } \\
(\mathrm{cm})\end{array}$ & $\begin{array}{c}\text { Mortalidad } \\
\%\end{array}$ & $\begin{array}{c}\text { Falla tardía } \\
\%\end{array}$ \\
\hline May $^{3}$ & 2001 & 148 & 99,0 & $3,9-8,0$ & 2,7 & 14,8 \\
Ouriel $^{16}$ & 2003 & 703 & 98,1 & $5,4 \pm 1,0$ & 1,7 & 15,0 \\
Prinssen $^{12}$ & 2004 & 171 & 97,8 & $6,1 \pm 0,9$ & 1,2 & 10,1 \\
EVAR 12 $^{22}$ & 2005 & 543 & 96,4 & $5,8-7,0$ & 1,6 & 15,0 \\
Serie UC & 2005 & 80 & 98,8 & $5,9 \pm 1,0$ & 1,3 & $6,3 *$ \\
\hline
\end{tabular}

*Incluye todos los pacientes aunque no hayan requerido tratamiento. 
La edad promedio de los pacientes en esta serie fue 73,6 años, casi 4 años mayor que los pacientes tratados en forma convencional durante el decenio 1986-199513, siendo frecuente la asociación de cardiopatía coronaria, hipertensión arterial y otras condiciones médicas que aumentan el riesgo operatorio (Tabla 3). En nuestro hospital, la mortalidad operatoria de la cirugía convencional del AAA es cercana al $1 \%^{13}$ coincidiendo con otros centros especializados ${ }^{14}$; en poblaciones de alto riesgo, como por ejemplo en pacientes octogenarios, la mortalidad llega a 5,4\%15. En series poblacionales no seleccionadas, que incluyen resultados de hospitales comunitarios y centros no especializados, la mortalidad operatoria alcanza a $4,2 \%{ }^{16}$. La gran ventaja de esta nueva técnica, tal como se puede corroborar en la presente serie, radica en una baja morbimortalidad operatoria, en una hospitalización y convalescencia más breves que la habitual para la cirugía convencional de la aorta abdominal, efectuada en un grupo etario particularmente vulnerable ${ }^{17,18}$.

El éxito y duración de la reparación endovascular del AAA se basa en la completa exclusión y depresurización del saco aneurismático, el que puede llegar a desaparecer (Figura 3). Si se produce una coaptación incompleta entre la endoprótesis y la zona no dilatada del vaso receptor, se puede generar una filtración entre la pared arterial y el dispositivo (endoleak o endofiltración» tipo I) presurizando el aneurisma, lo que hará fracasar su exclusión, por persistir la tensión sobre sus paredes. Lo mismo ocurre si la endoprótesis filtra por pérdida de su integridad o de la impermeabilidad de su pared (endofiltración tipo III y IV). Si ocurre dicha falla, no se previene la ruptura del AAA ${ }^{19}$. La endofiltración determinada por la persistencia de flujo revertido a través de ramas que nacen del saco aneurismático (endofiltración tipo II), no implica un aumento del riesgo de ruptura aórtica si no se acompaña de crecimiento progresivo del $\mathrm{AAA}^{20}$. En esta serie no hubo endofiltraciones precoces tipo I ni tipo III, y la tasa de endofiltración tipo II disminuyó espontáneamente con el tiempo, de 26,6\% en el posoperatorio inmediato a 7,6\%, según el último escáner de control. El único caso en que se evidenció crecimiento del AAA, se debió a una endofiltración tipo I a los 4 años, por migración distal de una endoprótesis Vanguard ${ }^{\circledR}$ de fijación infrarrenal, dispositivo posteriormente retirado del mercado por sus frecuentes complicaciones.

La evolución inmediata posterior al implante, se puede acompañar de un síndrome inflamatorio autolimitado, secundario a la trombosis del saco aneurismático y caracterizado por dolor dorso lumbar, alza térmica, leucocitosis, aumento de la VHS y de la proteína $C$ reactiva ${ }^{2}$. En la presente serie, dicho síndrome se presentó en casi la mitad de los pacientes y sólo requirió tratamiento sintomático.

En los casos técnicamente exitosos se observa una reducción gradual del tamaño del $\mathrm{AAA}^{21-24}$, fenómeno que varía según el tipo de endoprótesis utilizado ${ }^{21}$. En nuestra serie, 70,7\% de los pacientes presentó disminución del diámetro del AAA mayor a $5 \mathrm{~mm}$.

Tal como se puede apreciar en la Tabla 7, nuestros resultados son similares a los reportados desde otros países, destacando una menor tasa de complicaciones alejadas, que atribuimos a una mayor selectividad en la recomendación del procedimiento.

Actualmente, esta técnica innovadora es aplicable a más o menos la mitad de los pacientes estudiados por AAA. Aún no es posible utilizarla en forma universal por limitaciones anatómicas de muchos AAA, sin embargo, están en desarrollo complementos que permitirán aplicar este método a aneurismas de morfología más compleja ${ }^{25}$. Una segunda limitante de este procedimiento es su costo y la falta de cobertura adecuada por los aseguradores del sistema de salud. Si bien el ahorro en hospitalización y recuperación posoperatoria es importante, no es menos cierto que el costo del dispositivo y de la tecnología necesaria para aplicarlo y controlarlo posteriormente, actualmente supera el costo de la cirugía convencional ${ }^{26}$. Una tercera limitante es la dificultad de acceso a programas de entrenamiento acreditados y desarrollados en centros de alto volumen, que permitan la formación de especialistas que puedan aplicar esta técnica con seguridad para los pacientes.

La toma de decisiones respecto de la indicación de reparación convencional o endovascular, en nuestro criterio se debe basar, en primer lugar, en las características anatómicas del AAA. En segundo lugar, creemos recomendable reservar el 
uso de las endoprótesis preferentemente en los pacientes de mayor riesgo para la cirugía convencional (abdomen hostil y comorbilidades no subsanables), o con esperanza de vida menor de 5 años, considerando el incremento de la frecuencia de reintervenciones y por lo tanto mayores costos observados en el largo plazo ${ }^{27}$. Finalmente, la

\section{REFERENCIAS}

1. Parodi JC, Palmaz J, Barone HD. Transfemoral intraluminal graft inplantation for abdominal aortic aneurysms. Ann Vasc Surg 1991; 5: 491-9.

2. Blum U, Voshage G, Lammer J, Beyersdorf F, Tolner D, KRETSCHMER G ET AL. Endoluminal stent graft for infrarenal abdominal aortic aneurysms. N Eng J Med 1997; 336: 13-20.

3. May J, White GH, Waugh R, Ly CN, Stephen MS, JONES MA ET AL. Improved survival after endoluminal repair with second-generation prostheses compared with open repair in the treatment of abdominal aortic aneurysms: A 5-year concurrent comparison using life table method. J Vasc Surg 2001; 33 (Supplement): 21-6.

4. Valdés F, Seitz J, Fava M, Krämer A, Mertens R, Espíndola M et aL. Tratamiento del aneurisma aórtico abdominal por vía endovascular: Experiencia inicial. Rev Méd Chile 1998; 126: 1206-15.

5. Chaikoff EL, Blankenstejn JD, Harris PL, White $\mathrm{GH}$, Zarins CK, Bernhard VM et al. Reporting standards for endovascular aortic aneurysm repair. J Vacs Surg 2002; 35: 1048-60.

6. Valdés F, Sepúlveda N, Krämer A, Mertens $R$, Bergoeing M, Mariné L et al. Frecuencia de aneurisma aórtico abdominal en población adulta con factores de riesgo conocidos. Rev Méd Chile 2003; 131: 741-7.

7. Ingoldby CJ, Wujanto R, Mitchell JE. Impact of vascular surgery on community mortality from ruptured aortic aneurysms. Br J Surg 1986; 73: 551-3.

8. Lederle FA, Wilson SE, Johnson GR, ReinKe DB, LTTODY FM, ACHER CW ET AL. Immediate repair compared with surveillance of small abdominal aortic aneurysms. N Engl J Med 2002; 346: 1437-44.

9. The UK Small Aneury sm Trial Participants. Mortality results for randomized controlled trial of early optimización de la técnica y de los dispositivos a usar, la disminución de los costos, y el entrenamiento de especialistas en la aplicación de este sofisticado procedimiento, deberían tener un impacto positivo sobre su difusión, de modo de hacerlo más accesible a un mayor número de pacientes en centros debidamente capacitados.

elective surgery or ultrasonographic surveillance for small abdominal aortic aneurysms. Lancet 1998; 352: 1649-55.

10. Mertens R, Vaddés F, Kramer A. ¿Cuándo tratar pacientes con aneurismas aórticos abdominales de diámetro inferior a 5,5 cm? Rev Chil Cirugía 2004; 56: 510-1.

11. Brewster DC, Cronenwett JL, HaLett JW, Johnston KW, KRUPSKI WC, MATSUMURA JS. Guidelines for the treatment of abdominal aortic aneurysms: Report of a subcommittee of the Joint Council of the American Association for Vascular Surgery and Society for Vascular Surgery. J Vasc Surg 2003; 37: 1106-17.

12. THOMpson RW. Detection and management of small aortic aneurysms. N Engl J Med 2002; 346: 1484-6.

13. Valdés F, Krämer A, Mertens R, Santini A, Canessa R, LeMA G ET AL. Aneurisma aórtico abdominal: Evolución de la morbimortalidad de la cirugía electiva durante 20 años. Rev Méd Chile 1997; 125: 425-32.

14. Hertzer N, Mascha E, Karafa M, O'Hara P, KrajewsKI L, BEvEN E. Open infrarenal abdominal aortic aneurysm repair: The Cleveland Clinic experience from 1989 to 1998. J Vasc Surg 2002; 35: 114554.

15. Valdés F, Bergoeing M, Krämer A, Mertens R, Canessa R, Lema G et al. Aneurisma aórtico abdominal en pacientes mayores de 80 años: tratamiento quirúrgico convencional en 80 casos consecutivos. Rev Méd Chile 2003; 131: 981-6.

16. Huber TS, Wang JG, Derrow AE, Dame DA, Ozaki CK, ZeIENoCK GB. Experience in the United States with intact abdominal aortic aneurysm repair. J Vasc Surg 2001; 33: 304-11.

17. Prinssen M, Verhoeven ELG, Buth J, Philppe WM, CuYpers MD, MarC RHM et aL. A randomized trial comparing conventional and endovascular repair 
of abdominal aortic aneurysms. N Engl J Med 2004; 351: 1607-18.

18. Greenhalgh RM, Brown LC, Kwong GP, Powell JT, THOMPSON SG. Comparison of endovascular aneurysm repair with open repair in patients with abdominal aortic aneurysm (EVAR trial 1), 30-day operative mortality results: randomized controlled trial. Lancet 2004; 364: 843-8.

19. White GH, Yu W, May J, Chaufur X, Stephen MS. Endoleak as a complication of endoluminal grafting of abdominal aortic aneurysms: Classification, incidence, diagnosis and management. J Endovasc Surg 1997; 4: 152-68.

20. Resch T, Ivancev K, Lindh M, Nyman U, Brunkwall J, MaLne M et al. Persistent collateral perfusion of abdominal aortic aneurysm after endovascular repair does not lead to progressive change in aneurysm diameter. J Vasc Surg 1998; 28: 242-9.

21. Ouriel K, Ciair DG, Greenberg RK, Lyden SP, O’Hara PJ, Sarac TP ET al. Endovascular repair of abdominal aortic aneurysms: device-specific outcome. J Vasc Surg 2003; 37: 991-8.

22. May J, White GH, Yu W, Waugh RC, Stephen MS, HARRIS JP. A prospective study of changes in morphology and dimensions of abdominal aortic aneurysms following endoluminal repair. J Endovasc Surg 1995; 2: 343-7.

23. White RA, Donayre CE, Walot I, Kopchock GE, Wilson EP, Devirgilio C. Regression of an abdominal aortic aneurysm after endograft exclusion. J Vasc Surg 1997; 26: 133-7.

24. Wolf YG, HiL BB, Rubin GD, Fogarty TJ, Zarins $\mathrm{CK}$. Rate of change in abdominal aortic aneurysm diameter after endovascular repair. J Vasc Surg 2000; 32: 108-15.

25. Greenberg RK, Haulon S, Lyden SP, SRivastava SD, TuRC A, Eagleton MJ et al. Endovascular management of juxtarenal aneurysms with fenestrated endovascular grafting. J Vasc Surg 2004; 39: 279-87.

26. Angle N, Dorafshar AH, Moore WS, QuinonesBaldrich WJ, Gelabert HA, Ahn SS et al. Open versus endovascular repair of abdominal aortic aneurysms: what does each really cost? Ann Vasc Surg 2004; 18: 612-8.

27. EVAR TRIAL PARTICIPANTS. Endovascular aneurysm repair versus open repair in patients with abdominal aortic aneurysm (EVAR trial 1): randomized controlled trial. Lancet 2005; 365: 2179-86. 